\title{
Using nudges to promote healthy food choices at Greater Boston food pantries
}

\section{Abstract}

Nudging is a strategy used in behavioral economics to influence consumer decision-making through subtle changes in the choice environment. Recently, behavioral economists have been testing the use of nudge techniques to encourage healthier foods for high risk individuals. Food insecure individuals have an elevated risk of obesity and non-communicable diseases and would benefit from eating more nutrients dense foods for prevention and treatment. To promote more nutrient dense foods for food insecure individuals, Feeding America created a list of Foods to Encourage (F2E). This study evaluated the efficacy of nudge interventions in promoting two selected F2E at client-choice food pantries in Massachusetts. The objective of this study was to determine if the nudge interventions increased the take-rate of the targeted F2E: carrots and brown rice. Nudge interventions were implemented at three client-choice food pantries. Carrots were subjected to a "recipe nudge" in which recipe cards were placed next to the product. Brown rice underwent a placement nudge, which entailed moving the product to the proximal end of the display at waist height within easy reach and line of sight. Data was collected three times pre-intervention and three times post-intervention at each site to determine if the nudge had an impact on take rate. This study took place at three suburban food pantries in the Greater Boston area. The total number of shoppers observed before intervention was 402 and after intervention was 417 . The main outcome was the take-rate of the products determined by the number of shoppers who selected the foods before and after intervention. Analyses were performed using IBM SPSS Version 24 (Armonk, NY). Odds ratios were calculated to determine the effect of the intervention with a logistic regression controlling for the site. The take-rate of brown rice increased significantly post-placement intervention. The odds ratio for brown rice was $1.940(95 \% \mathrm{CI}$ $=1.318$ to 2.857). The take rate of other types of rice did not change post-intervention. The take rate for fresh carrots also increased significantly post recipe card intervention. The odds ratio for carrots was $1.519(95 \% \mathrm{CI}=1.129$ to 2.044$)$. The results of this study indicate a favorable effect of nudges on the take rate of nutrient dense products and could support the use of nudges as a strategy to promote the distribution of healthy food in client-choice food pantries.

\section{Conflict of Interest}

There is no conflict of interest. 